\title{
Proteinase 3 and neutrophil elastase enhance inflammation in mice by inactivating antiinflammatory progranulin
}

\author{
Kai Kessenbrock, ${ }^{1}$ Leopold Fröhlich, ${ }^{2}$ Michael Sixt, ${ }^{3}$ Tim Lämmermann, ${ }^{3}$ \\ Heiko Pfister, ${ }^{1}$ Andrew Bateman, ${ }^{4}$ Azzaq Belaaouaj, ${ }^{5}$ Johannes Ring, ${ }^{6,7}$ \\ Markus Ollert, ${ }^{6}$ Reinhard Fässler, ${ }^{3}$ and Dieter E. Jenne ${ }^{1}$
}

\begin{abstract}
${ }^{1}$ Department of Neuroimmunology, Max-Planck-Institute of Neurobiology, Martinsried, Germany. ${ }^{2}$ Institute of Pathophysiology, University of Veterinary Medicine Vienna, Vienna, Austria. ${ }^{3}$ Department of Molecular Medicine, Max-Planck-Institute of Biochemistry, Martinsried, Germany. ${ }^{4}$ Division of Experimental Medicine, McGill University and Endocrine Research Laboratory, Royal Victoria Hospital, Montreal, Quebec, Canada. 5INSERM UMR514, IFR 53, Hôpital Maison Blanche, CHU de Reims, Reims, France. 'Department of Dermatology and Allergy, Clinical Research Division of Molecular and Clinical Allergotoxicology, Technische Universität München, Munich, Germany. ${ }^{7}$ Division of Environmental Dermatology and Allergy, Helmoltz Zentrum München, German Research Center for Environmental Health, Neuherberg, Germany.
\end{abstract}

\begin{abstract}
Neutrophil granulocytes form the body's first line of antibacterial defense, but they also contribute to tissue injury and noninfectious, chronic inflammation. Proteinase 3 (PR3) and neutrophil elastase (NE) are 2 abundant neutrophil serine proteases implicated in antimicrobial defense with overlapping and potentially redundant substrate specificity. Here, we unraveled a cooperative role for PR3 and NE in neutrophil activation and noninfectious inflammation in vivo, which we believe to be novel. Mice lacking both PR3 and NE demonstrated strongly diminished immune complex-mediated (IC-mediated) neutrophil infiltration in vivo as well as reduced activation of isolated neutrophils by ICs in vitro. In contrast, in mice lacking just NE, neutrophil recruitment to ICs was only marginally impaired. The defects in mice lacking both PR3 and NE were directly linked to the accumulation of antiinflammatory progranulin (PGRN). Both PR3 and NE cleaved PGRN in vitro and during neutrophil activation and inflammation in vivo. Local administration of recombinant PGRN potently inhibited neutrophilic inflammation in vivo, demonstrating that PGRN represents a crucial inflammation-suppressing mediator. We conclude that PR3 and NE enhance neutrophil-dependent inflammation by eliminating the local antiinflammatory activity of PGRN. Our results support the use of serine protease inhibitors as antiinflammatory agents.
\end{abstract}

\section{Introduction}

Neutrophils belong to the body's first line of cellular defense and respond quickly to tissue injury and invading microorganisms (1). In a variety of human diseases, like autoimmune disorders, infections, or hypersensitivity reactions, the underlying pathogenic mechanism is the formation of antigen-antibody complexes, socalled immune complexes (ICs), which trigger an inflammatory response by inducing the infiltration of neutrophils (2). The subsequent stimulation of neutrophils by C3b-opsonized ICs results in the generation of ROS and the release of intracellularly stored proteases leading to tissue damage and inflammation (3). It is therefore important to identify the mechanisms that control the activation of infiltrating neutrophils.

Neutrophils abundantly express a unique set of neutrophil serine proteases (NSPs), namely cathepsin G (CG), proteinase 3 (PR3; encoded by Prtn3), and neutrophil elastase (NE; encoded by Ela2), which are stored in the cytoplasmic, azurophilic granules. PR3 and NE are closely related enzymes, with overlapping and potentially redundant substrate specificities different from

Nonstandard abbreviations used: CG, cathepsin G; EBM, extravascular basement membrane; GRN, granulin; HPF, high-power field; IC, immune complex; MPO, myeloperoxidase; NE, neutrophil elastase; NSP, neutrophil serine protease; PGRN, progranulin; phox, phagocyte oxidase; PR3, proteinase 3; RPA, reverse passive Arthus reaction.

Conflict of interest: The authors have declared that no conflict of interest exists. Citation for this article: J. Clin. Invest. 118:2438-2447 (2008). doi:10.1172/JCI34694. those of CG. All 3 NSPs are implicated in antimicrobial defense by degrading engulfed microorganisms inside the phagolysosomes of neutrophils (4-8). Among many other functions ascribed to these enzymes, PR3 and NE were also suggested to play a fundamental role in granulocyte development in the bone marrow (9-11).

While the vast majority of the enzymes is stored intracellularly, minor quantities of PR3 and NE are externalized early during neutrophil activation and remain bound to the cell surface, where they are protected against protease inhibitors $(12,13)$. These membrane presented proteases were suggested to act as path clearers for neutrophil migration by degrading components of the extracellular matrix (14). This notion has been addressed in a number of studies, which yielded conflicting results (15-17). Thus, the role of PR3 and NE in leukocyte extravasation and interstitial migration still remains controversial.

Emerging data suggest that externalized NSPs can contribute to inflammatory processes in a more complex way than by simple proteolytic tissue degradation (18). For instance, recent observations using mice double-deficient for CG and NE indicate that pericellular CG enhances IC-mediated neutrophil activation and inflammation by modulating integrin clustering on the neutrophil cell surface $(19,20)$. Because to our knowledge no Prtn3 ${ }^{-}$ mice have previously been generated, the role of this NSP in inflammatory processes has not been deciphered. Moreover, NEdependent functions that can be compensated by PR3 in Ela2animals are still elusive. 


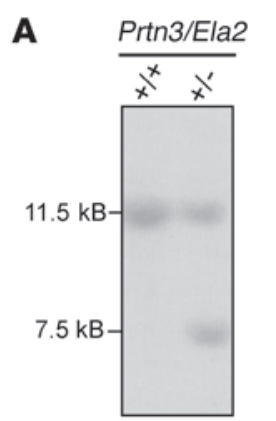

E

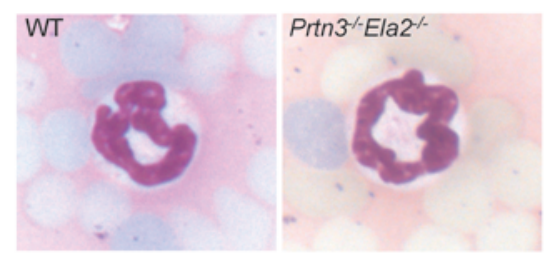

C

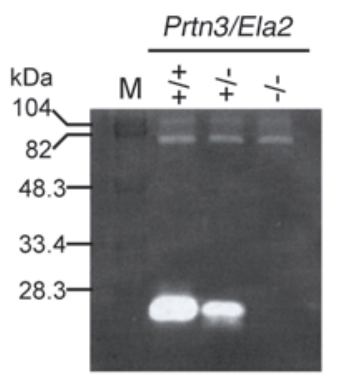

$\mathbf{F}$

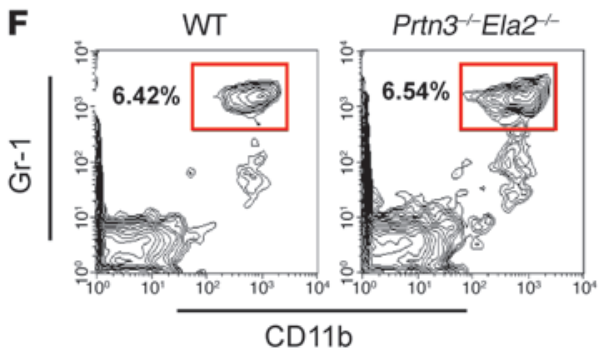

D
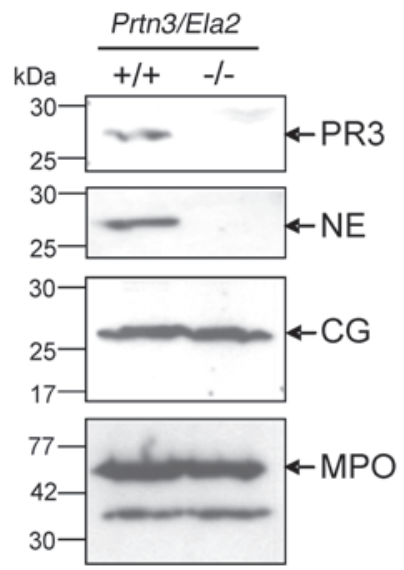

Figure 1

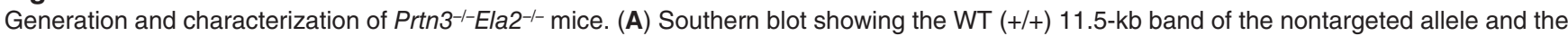
presence of an additional 7.5-kb band in a heterozygous (+/-) PR3/NE-targeted embryonic stem cell clone. (B) RT-PCR revealed the complete lack of mouse PR3 (mPR3; $437 \mathrm{bp}$ ) and mouse NE (743 bp) transcripts in bone marrow cells from Prtn3 $3^{-/-}$Ela2 $2^{-/-}$mice $(-/-)$, while expression of $\beta$-actin (699 bp) was normal. (C) Casein zymography showed prominent casein degradative activity at $27 \mathrm{kDa}$ in WT neutrophil lysates, while intermediate degradation by heterozygous lines and no degradation by Prtn3 $3^{-/} E l a 2^{-/-}$lines was found at this size. M, marker. (D) Western blot

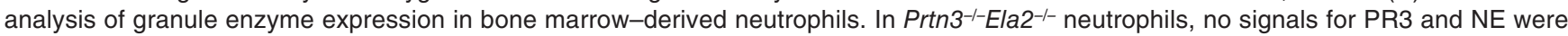
detected, while CG ( $\sim 26 \mathrm{kDa})$, MPO ( $\sim 59 \mathrm{kDa})$, and a smaller degradation product of MPO were detected at the same levels as in WT neutro-

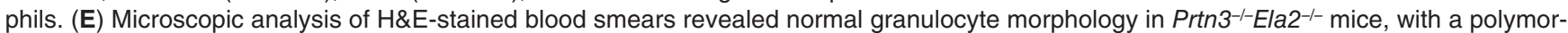
phic nucleus (dark blue) identical to that of WT neutrophils. Original magnification, $\times 20$. (F) Flow cytometry of peripheral blood with gating on $\mathrm{Gr}-1^{\text {hi }} \mathrm{CD} 11 \mathrm{~b}^{+}$showed regular neutrophil populations (boxed regions) in Prtn3 ${ }^{-1-E} / \mathrm{a} 2^{-/-}$mice. Plots are representative of data obtained from 3 mice per group. Percentages denote percent cells in the boxed regions.

One mechanism by which NSPs could upregulate the inflammatory response has recently been proposed. The ubiquitously expressed progranulin (PGRN) is a growth factor implicated in tissue regeneration, tumorigenesis, and inflammation (21-23). PGRN was previously shown to directly inhibit adhesion-dependent neutrophil activation by suppressing the production of ROS and the release of neutrophil proteases in vitro (23). This antiinflammatory activity was degraded by NE-mediated proteolysis of PGRN to granulin (GRN) peptides (23). In contrast, GRN peptides may enhance inflammation (23) and have been detected in neutrophil-rich peritoneal exudates (24). In short, recent studies proposed PGRN as a regulator of the innate immune response, but the factors that control PGRN function are still poorly defined and its relevance to inflammation needs to be elucidated in vivo.

In the present study, we generated double-deficient $\operatorname{Prtn} 3^{-/-}$ Ela2 $2^{-1-}$ mice to investigate the role of these highly similar serine proteases in noninfectious neutrophilic inflammation. We established that PR3 and NE are required for acute inflammation in response to subcutaneous IC formation. The proteases were found to be directly involved in early neutrophil activation events, because isolated $\operatorname{Prtn}^{-/-} \mathrm{Ela2}^{-/-}$neutrophils were poorly activated by ICs in vitro. These defects in Prtn3 $3^{-/-}$Ela2 $2^{-/-}$mice were accompanied by accumulation of PGRN. We demonstrated that PGRN represents a potent inflammation-suppressing factor that is cleaved by both PR3 and NE. Our data delineate what we believe to be a previously unknown proinflammatory role for PR3 and NE, which is accomplished via the local inactivation of antiinflammatory PGRN.

\section{Results}

Generation of Prtn $3^{-/-}$Ela2 $2^{-/}$mice. To analyze the role of PR3 and NE in neutrophilic inflammation, we generated a $\operatorname{Prtn}^{-/-}$Ela $2^{-1-}$ mouse line by targeted gene disruption in embryonic stem cells (see Supplemental Figure 1; supplemental material available online with this article; doi:10.1172/JCI34694DS1). Positive recombination of the Prtn3/Ela2 locus was proven by Southern blotting of embryonic stem cell clones (Figure 1A). Prtn $3^{-/-}$Ela2 ${ }^{-/-}$mice showed no expression of mRNA for PR3 and NE in bone marrow cells, as assessed by RT-PCR (Figure 1B). The successful elimination of PR3 and NE was confirmed at the level of proteolytic activity in neutrophil lysates using a PR3/NE-specific chromogenic substrate (Supplemental Figure 3) as well as by casein zymography (Figure 1C). The substantially reduced casein degradation by heterozygous neutrophils indicates gene-dosage dependence of PR3/NE activities. Furthermore, PR3 and NE deficiency was proven by Western blotting using cell lysates from bone marrow-derived neutrophils, while other enzymes stored in azurophilic granula, such as CG and myeloperoxidase (MPO), were normally detected (Figure 1D). Crossing of heterozygous $\operatorname{Prtn}^{+/-} \mathrm{Ela2}^{+/-}$mice resulted in regular offspring of WT, heterozygous, and homozygous genotype according to the Mendelian ratio. Despite the absence of 2 abundant serine proteases, and in contrast to expectations based on previous reports (9-11), we found unchanged neutrophil morphology (Figure $1 \mathrm{E})$ and regular neutrophil populations in the peripheral blood of the mutant mice, the latter as assessed via flow cytometry to determine the differentiation markers CD11b and Gr-1 (Figure 1F) 
A
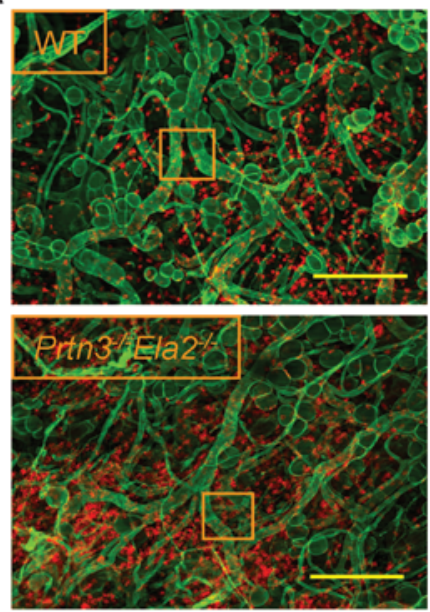

D

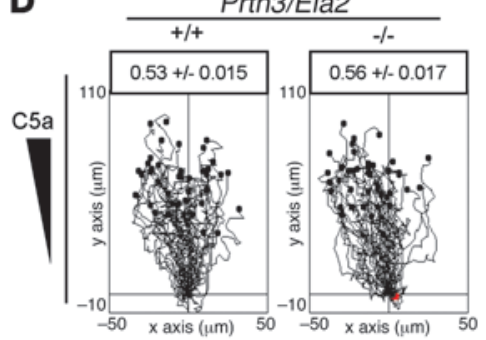

B
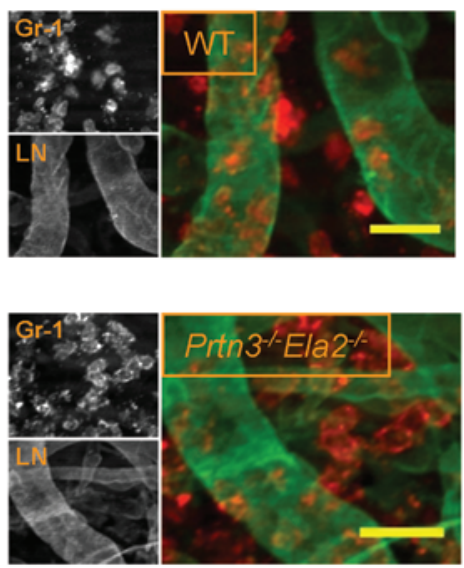

E

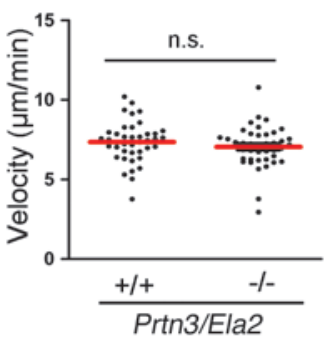

C

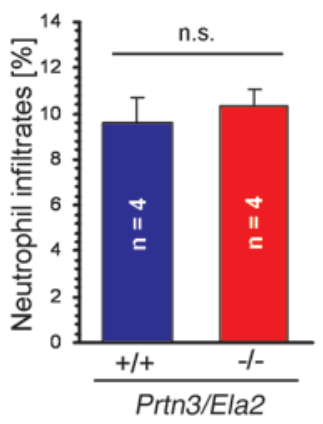

\section{Figure 2}

PR3 and NE are not principally required for neutrophil extravasation and interstitial migration. (A and B) Phorbol ester-treated ear tissues of WT and Prtn3 ${ }^{-/-E l a 2} 2^{-/-}$mice were immunostained for laminin (LN; green) to visualize EBM and Gr-1 (red) to identify neutrophils. Lesions were examined $4 \mathrm{~h}$ after stimulus application by fluorescence microscopy as described in Methods. (A) Representative images of tissue from WT and Prtn3---Ela2-/- mice. Both genotypes developed strong and widespread neutrophil infiltrations. (B) Higher-magnification images of boxed regions in A. Prtn3 ${ }^{--}$Ela2 ${ }^{-/-}$neutrophils showed no retention at the EBM. Scale bars: $200 \mu \mathrm{m}$ (A); $25 \mu \mathrm{m}$ (B). (C) Overall neutrophil infiltrates were quantified as the percentage of $\mathrm{Gr}-1-$ positive cells per microscopic field. Data are mean \pm SEM. Intravascular cells were excluded. No significant differ-

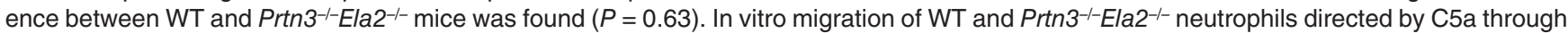
3-dimensional collagen matrices was analyzed by time-lapse video microscopy (see Supplemental Video 1). (D) The tracks of WT ( $n=41$ ) and Prtn3 $^{---}$Ela2 $^{--}(n=42)$ neutrophils are shown, and the factor for directionality \pm SEM is indicated. No impairment was observed regarding chemotactic directionality of $P r t n 3^{--}$Ela2 ${ }^{--}$versus WT neutrophils $(P=0.19)$. (E) Velocities of single cells (individual points) were calculated and averaged (red bar). Prtn3---Ela2-/- neutrophils showed no significant difference versus WT cells $(P=0.30)$.

$(25,26)$. Moreover, Prtn3 $3^{-/-}$Ela2 $2^{-/-}$mice demonstrated normal percentages of the leukocyte subpopulations in the peripheral blood, as determined by the Diff-Quick staining protocol and by hemocytometric counting (Supplemental Figure 2, A and B). Hence, the proteases are not crucially involved in granulopoiesis, and ablating PR3 and NE in the germ line represents a valid approach to assess their biological significance in vivo.

$P R 3$ and NE are dispensable for neutrophil extravasation and interstitial migration. To examine neutrophil infiltration into the perivascular tissue, we applied phorbol esters (croton oil) to the mouse ears. At $4 \mathrm{~h}$ after stimulation, we assessed the neutrophil distribution in relation to the extravascular basement membrane (EBM) by immunofluorescence microscopy of fixed whole-mount specimens (Figure 2A). We found that $\operatorname{Prtn}^{-/-} \mathrm{Ela}^{-/-}$neutrophils transmigrated into the interstitium without retention at the EBM (Figure 2B), resulting in quantitatively normal and widespread neutrophil influx compared with WT mice (Figure 2C). Moreover, we analyzed chemotactic migration of isolated neutrophils through a 3 -dimensional collagen meshwork in vitro (Supple- mental Video 1) and found unhampered chemotaxis toward a C5a gradient, based on the directionality (Figure 2D) and velocity (Figure 2E) of Prtn3 $3^{-/-} \mathrm{Ela}^{-{ }^{--}}$neutrophils. These findings led us to conclude that PR3 and NE are not principally required for neutrophil extravasation or interstitial migration.

Reduced inflammatory response to ICs in Prtn $3^{-1-} \mathrm{Ela2}^{-1-}$ mice. The formation of ICs represents an important trigger of neutrophildependent inflammation in many human diseases (2). To determine the role of PR3 and NE in this context, we induced a classic model of subcutaneous IC-mediated inflammation, namely the reverse passive Arthus reaction (RPA) (27). At $4 \mathrm{~h}$ after RPA induction, we assessed the cellular inflammatory infiltrates by histology using H\&E-stained skin sections (Figure 3A). Neutrophils, which were additionally identified by Gr-1 immunohistochemistry, made up the vast majority of all cellular infiltrates (Figure 3A). We found that neutrophil infiltration to the sites of IC formation was severely diminished in Prtn $3^{-/-}$Ela $^{-/-}$mice. Indeed, histological quantification revealed significantly reduced neutrophil influx in Prtn $3^{-/-}$Ela2 $2^{-/-}$mice compared with WT mice, while Ela2 $2^{-/}$mice 
A

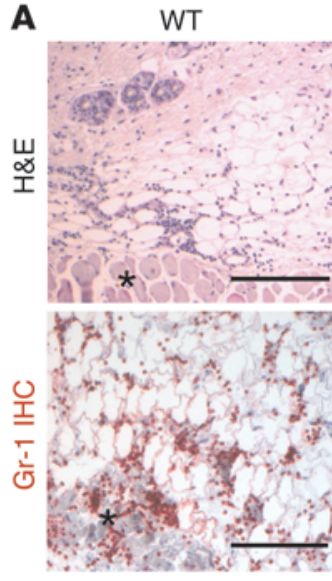

Ela2-

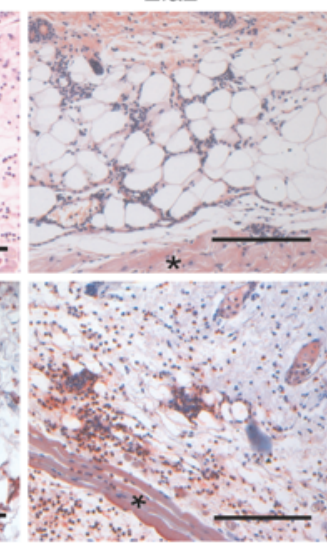

Prtn3'-Ela2--

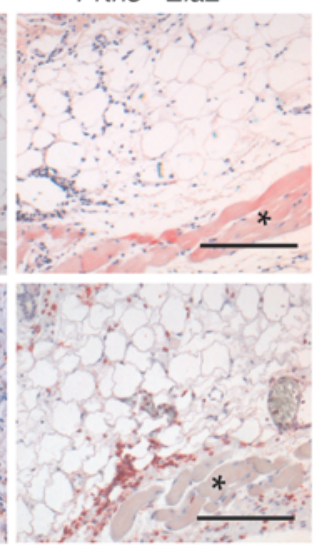

B

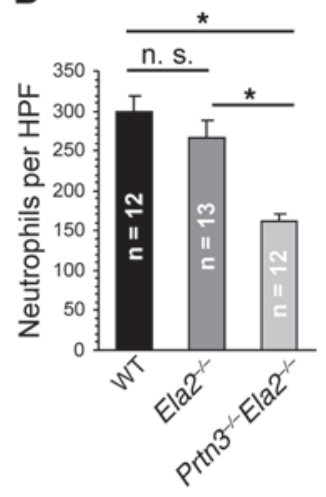

\section{Figure 3}

Impaired inflammatory response to locally formed ICs in Prtn3--Ela2 ${ }^{-/-}$mice. (A) Representative photomicrographs of inflamed skin sections $4 \mathrm{~h}$ after IC formation. Neutrophils were identified morphologically (polymorphic nucleus) in H\&E stainings and by Gr-1 staining (red). The cellular infiltrates were located to the adipose tissue next to the panniculus carnosus muscle (asterisks) and were primarily composed of neutrophil

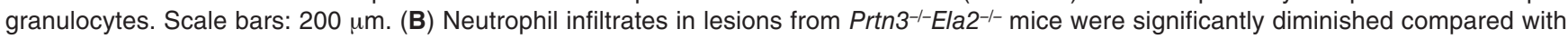
Ela2 ${ }^{-/}$mice and WT mice. Neutrophil influx in Ela2 ${ }^{--}$mice was slightly, but not significantly, diminished compared with WT mice. Results are mean \pm SEM infiltrated neutrophils per HPF. ${ }^{*} P<0.05$.

showed marginally reduced neutrophil counts (Figure 3B). These results indicate that PR3 and NE fulfill an important proinflammatory function during IC-mediated inflammation.

$P R 3$ and NE enhance neutrophil activation by ICs in vitro. Because PR3 and NE were required for the inflammatory response to IC (Figure 3 ), but not to phorbol esters (Figure 2), we considered the enzymes as enhancers of the neutrophil response to IC. We therefore assessed the oxidative burst using dihydrorhodamine as a readout for cellular activation of isolated, TNF- $\alpha$-primed neutrophils in the presence of

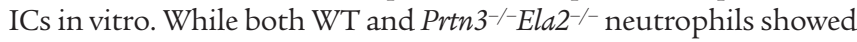
a similar, approximately 20 -min lag phase before the oxidative burst commenced, the ROS production over time was markedly reduced, by $30 \%-40 \%$, in the absence of PR 3 and NE (Figure $4 \mathrm{~A}$ ). In contrast, oxidative burst triggered by $25 \mathrm{nM}$ PMA was not hindered in Prtn $3^{-/-}$ Ela2 ${ }^{-/}$neutrophils (Figure 4B), which indicated no general defect in producing ROS. We also performed a titration series ranging from 0.1 to $50 \mathrm{nM}$ PMA and found no reduction in oxidative burst activity in $\operatorname{Prtn} 3^{-/-}$Ela2 $2^{-/}$neutrophils at any PMA concentration used (Supplemental Figure 4). These data are consistent with our in vivo experiments showing that neutrophil influx to ICs was impaired (Figure 3), whereas the inflammatory response to phorbol esters was normal (Figure 2, A-C), in Prtn3 3-Ela2-/- mice. To compare neutrophil priming in WT and $\mathrm{Prtn}^{-/-}$Ela $2^{-/-}$neutrophils, we analyzed cell surface expression of $\mathrm{CD} 11 \mathrm{~b}$ after $30 \mathrm{~min}$ of incubation at various concentrations of TNF- $\alpha$ and found no difference (Supplemental Figure 5). Moreover, we observed normal neutrophil adhesion to ICcoated surfaces (Supplemental Figure 6A) and unaltered phagocytosis of opsonized, fluorescently labeled E. coli bacteria (Supplemental Figure 6, B and C) in the absence of both proteases. We therefore hypothesized that PR3 and NE enhance early events of adhesiondependent neutrophil activation after TNF- $\alpha$ priming and binding of ICs. It is important to note that Ela2 $2^{--}$neutrophils were previously shown to react normally in the same setup (20). Regarding the highly similar cleavage specificities of both proteases, we suggested that PR3 and NE complemented each other during the process of neutrophil activation and inflammation.
Antiinflammatory PGRN is degraded by PR3 and NE during IC-mediated neutrophil activation. This reasoning prompted us to search for substrates that are processed by both PR3 and NE and are thereby able to enhance neutrophilic activation. In a previous study, PGRN was described as a potent inhibitor of the adhesion-dependent oxidative burst of human TNF- $\alpha$-primed neutrophils in vitro, which can be inactivated by NE (23). Because we observed a substantial reduction in ROS production in Prtn3 $3^{-/}$Ela2 $2^{-/}$neutrophils compared with WT cells (Figure 4A), we evaluated a potential link between PGRN cleavage and oxidative burst of neutrophils activated by ICs. We analyzed the culture supernatant as well as the cellular pellet of IC-activated neutrophils for the presence of PGRN by IB (Figure 4C). Indeed, the inhibitory, intact form of PGRN was detected in the supernatant of $\operatorname{Prtn}^{-/-}$Ela2 $2^{-/}$neutrophils only. We also found a cellular pool of PGRN in all genotypes that was more abundant in Prtn $3^{-/-}$Ela2 $2^{-/}$cells than in WT or Ela2 $2^{-/-}$neutrophils. This led us to conclude that PGRN was released and degraded by PR3 and NE during neutrophil activation.

PGRN inbibits neutrophil activation by ICs in vitro. To show that the reduced oxidative burst of $\mathrm{Prtn}^{-/-}$-Ela2 $2^{-/-}$neutrophils can be caused by defective PGRN degradation, we evaluated the amount of cellassociated and secreted PGRN detected by IB (Figure 4C). We tested whether these concentrations inhibited the IC-mediated oxidative burst of WT neutrophils when added to the cells in vitro. Indeed, when $100 \mathrm{nM}$ PGRN was applied to WT granulocytes activated by ICs, the ROS response markedly decreased (Figure 4D). In positive control experiments, activation of neutrophils was unaffected by PGRN application when they were stimulated with PMA (Figure 4E). These data endorse the inhibitory effect of intact PGRN on innate immune cell activation and prove that defective PGRN degradation may cause the reduced activation and oxidative burst of Prtn3 $3^{--}$Ela2 $2^{--}$neutrophils triggered by ICs.

Both PR3 and NE process PGRN in vitro. In previous studies, NE was proposed as the principal PGRN-converting protease (23). Our experiments revealed an equally important role of PR 3 in this pathway, because Ela2-- neutrophils were not impaired in 
A

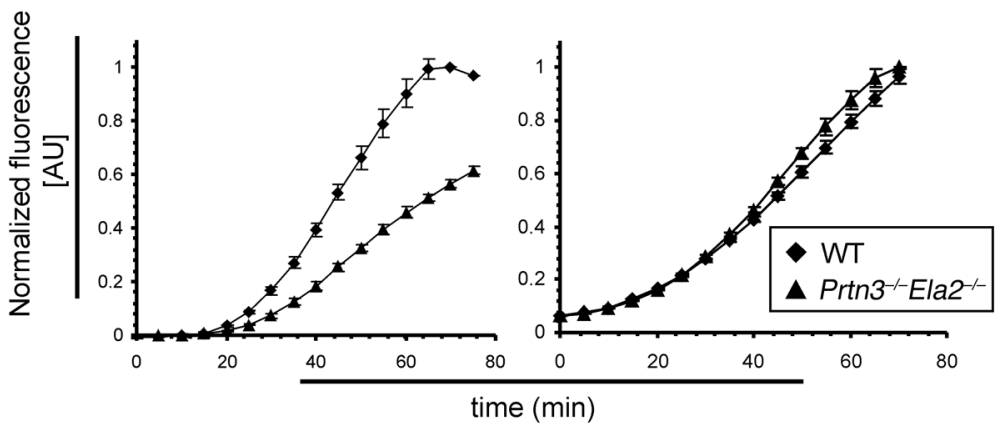

D

E

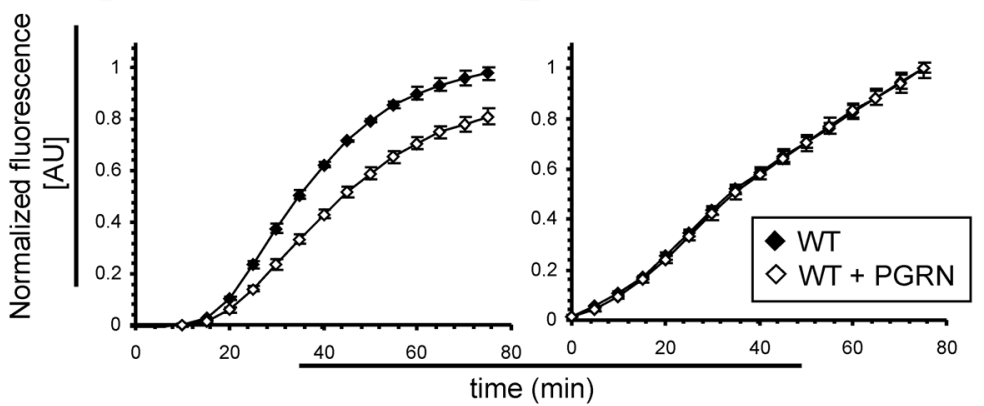

C

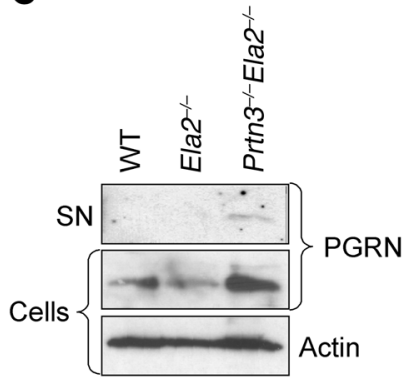

Figure 4

Impaired oxidative burst and PGRN degradation by IC-activated Prtn3 $3^{--E}$ Ela2 ${ }^{-/-}$neutrophils. Oxidative burst as the readout for neutrophil activation by ICs was measured over time. (A) While no difference was observed during the initial 20-min lag phase of the oxidative burst, Prtn3 ${ }^{-/-}$ Ela2 $^{-/-}$neutrophils exhibited diminished ROS production over time compared with WT neutrophils. (B) Bypassing receptor-mediated activation

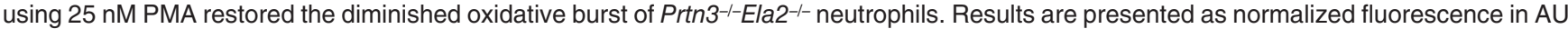
(relative to maximum fluorescence produced by WT cells). Data (mean \pm SD) are representative of 3 independent experiments each conducted in triplicate. (C) Isolated mouse neutrophils were activated by ICs in vitro and tested for PGRN degradation by IB. In the cellular fraction, the

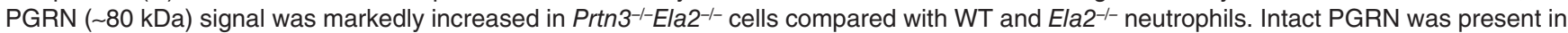
the supernatant (SN) of IC-activated Prtn3 ${ }^{-/-}$Ela2 $2^{-/}$neutrophils only, not of WT or Ela2 ${ }^{-/-}$cells. (D and E) Exogenous administration of 100 nM PGRN significantly reduced ROS production of neutrophils activated by ICs (D), but not when activated by PMA (E). Data (mean \pm SD) are representative of 3 independent experiments each conducted in triplicate.

their degradation of PGRN (Figure 4C). We further substantiated this concept by incubating recombinant PGRN with the purified proteases in vitro. Both enzymes completely degraded PGRN as early as 5 min after incubation, although the pattern of lowermolecular weight cleavage products analyzed by silver-stained SDS-PAGE was not identical for both proteases (Figure 5, A and B). Hence, both PR3 and NE are potent converters of PGRN, indicating biological redundancy of the proteases in this process.

$P R 3$ and NE are major PGRN-degrading enzymes of neutrophils. To evaluate the significance of PR3 and NE as PGRN-degrading enzymes, we next incubated recombinant mouse PGRN with neutrophil lysates from WT and protease-deficient mice. Anti-PGRN Western blot revealed that PGRN degradation was minimally reduced in the absence of NE, but strongly impaired when both PR3 and NE were lacking (Figure 5C). This observation demonstrates that PR3 and $\mathrm{NE}$ are major PGRN-converting enzymes of neutrophils, a finding supported by previous experiments showing that CG, the third NSP of primary granules, does not cleave PGRN (23).

$P G R N$ inhibits IC-mediated inflammation in vivo. To provide in vivo evidence for the relevance of PGRN as an antiinflammatory mediator, we administered $2 \mu \mathrm{g}$ recombinant PGRN to IC-mediated inflammation in mice. PGRN-treated lesions were directly compared with untreated lesions in the same mouse (Figure 6A). In both WT and Prtn3 ${ }^{-1-}$ Ela2 $2^{-1-}$ mice, neutrophil accumulation was diminished at the
PGRN-treated sites of IC-mediated inflammation, demonstrating that PGRN is a crucial inhibitory factor for neutrophilic inflammation. Neutrophil infiltration was reduced to a greater extent in Prtn $^{-/-}$Ela2 $^{-/-}$(40\% reduction, $P<0.01$; Figure 6C) than in WT mice ( $25 \%$ reduction, $P<0.05$; Figure $6 \mathrm{~B}$ ), which likely reflected the impact of the PGRN-degrading proteases in this pathway.

$P R 3$ and NE cleave PGRN during inflammation in vivo. Finally, we aimed to demonstrate defective PGRN degradation in Prtn $3^{-/-}$Ela2 $^{-/-}$ mice during neutrophilic inflammation in vivo. For practical reasons, we harvested infiltrated neutrophils from the inflamed peritoneum $4 \mathrm{~h}$ after casein injection and subjected the lysates of these cells to anti-PGRN Western blot. Intact, inhibitory PGRN was detected in Prtn3 $3^{-/-}$Ela2 $2^{-/-}$neutrophils, but not in WT cells (Figure 6D). These data prove that neutrophilic inflammation is accompanied by proteolytic removal of antiinflammatory PGRN and that the process of PGRN degradation is essentially impaired in vivo in the absence of PR3 and NE.

\section{Discussion}

Chronic inflammatory and autoimmune diseases are often perpetuated by continuous neutrophil infiltration and activation. According to the current view, the role of NSPs in these diseases is mainly associated with proteolytic tissue degradation after their release from activated or dying neutrophils. However, recent obser- 
A

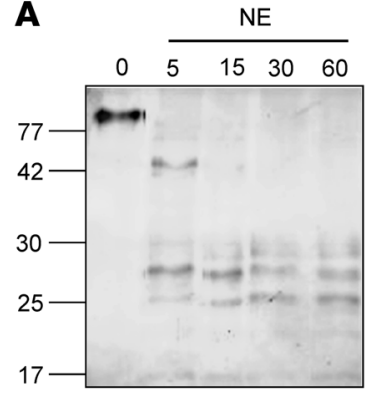

B

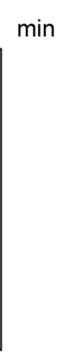

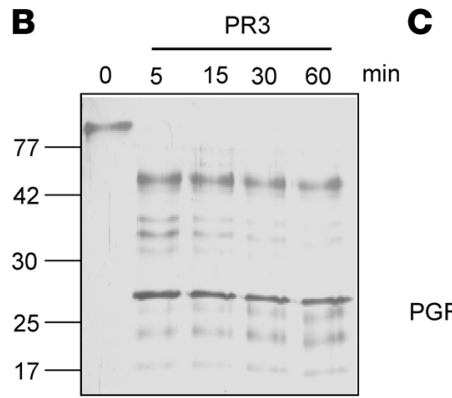

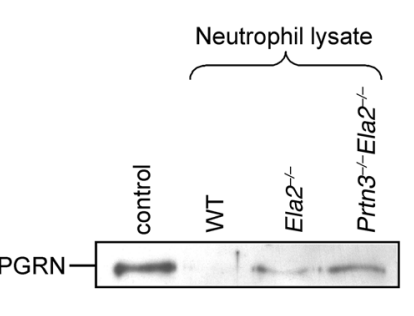

Figure 5

PR3 and NE are major PGRN processing enzymes of neutrophils. (A and B) Silver-stained SDS-PAGE analysis of recombinant human PGRN incubated at a 1:10 enzyme/substrate ratio with purified human NE (A) and recombinant mouse PR3 (B). Both NE and PR3 completely cleaved $\sim 80-k D a$ PGRN to smaller molecular fragments within 5 min of incubation. (C) Recombinant mouse PGRN was incubated with neutrophil lysates from WT, Ela2 ${ }^{-/-}$, and $\mathrm{Prtn}^{-/-}$Ela2 $2^{-/}$mice for $1 \mathrm{~h}$ at $37^{\circ} \mathrm{C}$ and analyzed by anti-mouse PGRN Western blot. Compared with untreated PGRN (control), WT neutrophils completely degraded

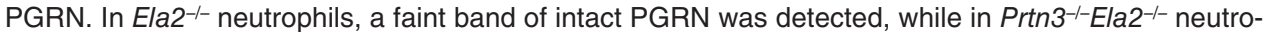
phils, a distinct PGRN band remained, comparable to control.

vations suggest that NSPs such as CG may contribute to noninfectious diseases in a more complex manner, namely as specific regulators of inflammation (18). Here, we demonstrate that PR3 and NE cooperatively fulfilled an important proinflammatory role during neutrophilic inflammation. PR3 and NE directly enhanced neutrophil activation by degrading oxidative burst-suppressing PGRN. These findings support the use of specific serine protease inhibitors as antiinflammatory agents.

Much attention has been paid to the degradation of extracellular matrix components by NSPs. We therefore expected that ablation of both PR3 and NE would cause impaired neutrophil extravasation and interstitial migration. Surprisingly, we found that the proteases were principally dispensable for these processes: Prtn $3^{-/-}$Ela2 ${ }^{-/-}$neutrophils migrated normally through a dense, 3 -dimensional collagen matrix in vitro and demonstrated regular extravasation in vivo when phorbol esters were applied (Figure 2). This finding is in agreement with recent reports that neutrophils preferentially and readily cross the EBM through regions of low matrix density in the absence of NE (28).

Conversely, we observed that PR3 and NE were required for the inflammatory response to locally formed ICs (Figure 3). Even iso-

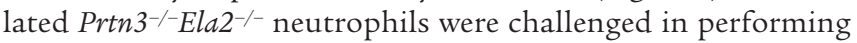
oxidative burst after IC stimulation in vitro (Figure 4A), showing that the proteases directly enhanced the activation of neutrophils also in the absence of extracellular matrix. However, when receptor-mediated signal transduction was bypassed by means of PMA, neutrophils from $\operatorname{Prtn}^{-1-}$ Ela $^{-1-}$ mice performed normal oxidative burst (Figure 4B), indicating that the function of the phagocyte oxidase (phox) complex was not altered in the absence of PR3 and NE. These findings substantiate what we believe to be a novel paradigm: that all 3 serine proteases of azurophilic granules (CG, PR3, and NE), after their release in response to IC encounter, potentiate a positive autocrine feedback on neutrophil activation.

In contrast to CG, the highly related proteases PR3 and NE cooperate in the effacement of antiinflammatory PGRN, leading to enhanced neutrophil activation. Previous studies already demonstrated that PGRN is a potent inhibitor of the adhesion-dependent oxidative burst of neutrophils in vitro, which can be degraded by NE (23). Here, we showed that PR3 and NE play an equally important role
The molecular mechanism of PGRN function is not yet completely understood, but it seems to interfere with integrin (CD11b/CD18) outside-in signaling by blocking the function of $\mathrm{pyk} 2$ and thus dampens adhesion-related oxidative burst even when added after the initial lag phase of oxidase activation (23). PGRN is produced by neutrophils and stored in highly mobile secretory granules (29). It was recently shown that PGRN can bind to heparan-sulfated proteoglycans (30), which are abundant components of the EBM and various cell surfaces, including those of neutrophils. Also, PR3 and NE are known to interact with heparan sulfates on the outer membrane of neutrophils, where the enzymes appear to be protected against protease inhibitors $(12,13,31)$. These circumstantial observations support the notion that PGRN cleavage by PR3 and NE takes place at the pericellular microenvironment of the neutrophil cell surface.

Impaired outside-in signaling most likely reduced the oxidative burst in Prtn $3^{-/-}$Ela2 $2^{-/}$neutrophils adhering to ICs. In support of this hypothesis, we excluded an altered response to TNF- $\alpha$ priming (Supplemental Figure 5) as well as reduced adhesion to immobilized ICs and defective endocytosis of serum-opsonized E. coli in Prtn3 ${ }^{-/-}$Ela2 $^{-/-}$neutrophils (Supplemental Figure 6). MPO content and processing was also unchanged in $\operatorname{Prtn}^{-/-}$Ela2 $^{-/-}$neutrophils (Figure 1D); hence, the previously discussed inhibitory effect of MPO on phox activity $(32,33)$ does not appear to be stronger in neutrophils lacking PR3 and NE. Because there was no difference in the lag phase of the oxidative burst, initial IC-triggered receptor activation was probably not affected by either PRGN or PR3/NE. Our concept is consistent with all these observations and takes into account that PGRN unfolds its suppressing effects in the second phase, when additional membrane receptors, endogenous PGRN, and some PR3/NE from highly mobile intracellular pools are translocated to the cell surface. The decline and cessation of ROS production suggested to us that outside-in signaling was not sustained and that active oxidase complexes were no longer replenished in the absence of PR3 and NE. Our present findings, however, do not allow us to exclude other potential mechanisms, such as accelerated disassembly of the active oxidase complex.

During cutaneous inflammation, PGRN is provided by multiple sources, including skin cells and neutrophils themselves, which produce and release PGRN as they infiltrate the tissue $(21,29)$. 
A

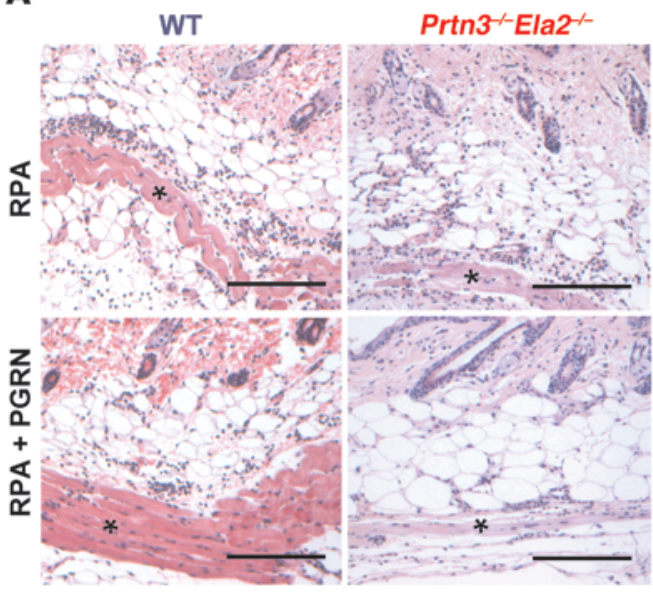

B

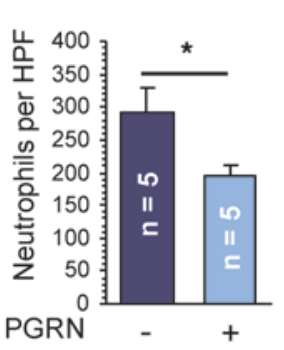

C

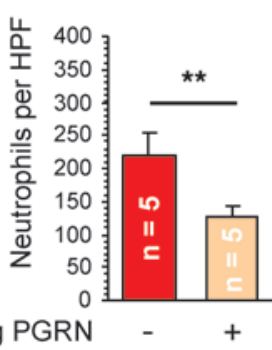

D

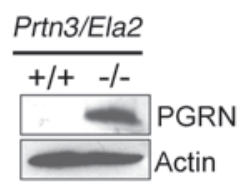

\section{Figure 6}

PGRN is a potent inhibitor of IC-stimulated inflammation in vivo. Recombinant mouse PGRN (2 $\mu \mathrm{g})$ was intradermally applied with anti-OVA IgG, and the RPA was started in WT and Prtn3 ${ }^{--} E l a 2^{--}$mice $(n=5$ per group). (A) After $4 \mathrm{~h}$, the effect of PGRN application was evaluated by histological analyses. Representative images show neutrophil infiltrates at the panniculus carnosus muscle (asterisks). Scale bars: $200 \mu \mathrm{m}$. (B and C) Effect of PGRN administration on neutrophil influx. In both WT (B) and Prtn3-/-Ela2-/- (C) mice, neutrophil infiltration was significantly diminished at PGRN-treated sites compared with untreated sites. This effect appeared to be more pronounced in the protease-deficient mice. Data are mean \pm SEM infiltrated neutrophils per HPF. ${ }^{*} P<0.05 ;{ }^{* *} P<0.01$. (D) Neutrophils isolated ex vivo from inflamed peritoneum of WT and Prtn3 ${ }^{-1-E l a 2^{-/}}$mice were analyzed by anti-mouse PGRN Western blot of concentrated neutrophil lysates. Intact PGRN was found abundantly in Prtn3 ${ }^{-1-E l a 2}{ }^{--}$but not WT neutrophils. Loading was controlled using anti-actin Western blot.

Thus, PGRN represents a prominent factor to control extravascular neutrophil function during skin inflammation. Proteolytic processing of PGRN was previously shown to generate GRN peptides that accumulate in inflammatory exudates, such as during caseininduced peritonitis (34). In contrast to the precursor PGRN, these GRN peptides might be proinflammatory and induce the release of neutrophil-attracting IL-8 from epithelial cells (23). In the absence of PR3 and NE, neutrophils are no longer able to provide the switch from inflammation-suppressing PGRN to proinflammatory GRN peptides. The activation of neutrophils by ICs is a decisive event in the cascade of reactions during the RPA. IC-activated neutrophils are known to release cytokines and chemokines and thus establish an inflammatory milieu. We hypothesize that the local persistence of PGRN dampens neutrophil activation and release of ROS as well as other inflammatory mediators in response to ICs, which results in diminished recruitment of further neutrophils in $\mathrm{Prtn}^{-/-} \mathrm{Ela2}^{-/-}$ mice. Hence, defective PGRN degradation can account for the impaired inflammation in the absence of PR3 and NE (Figure 7).

NSPs are strongly implicated as effector molecules in a large number of destructive diseases, such as emphysema or the autoimmune blistering skin disease bullous pemphigoid (14, 35-37). Normally, PR3/NE activity is tightly controlled by high plasma levels of $\alpha 1$-antitrypsin. This balance between proteases and protease inhibitors is disrupted in patients with genetic $\alpha 1$-antitrypsin deficiency, which represents a high risk factor for the development of emphysema and certain autoimmune disorders (38). The pathogenic effects of NSPs in these diseases have so far been associated with tissue destruction by the proteases after their release from dying neutrophils. Our findings showed that PR3 and NE were already involved in much earlier events of the inflammatory process, because the enzymes directly regulated cellular activation of infiltrating neutrophils by degrading inflammation-suppressing PGRN. This concept is further supported by previous studies showing increased inflammation in mice lacking serine protease inhibitors such as SERPINB1 or SLPI $(39,40)$. Blocking PR3/NE activity using specific inhibitors therefore represents a promising therapeutic strategy to treat chronic, noninfectious inflammation. Serine protease inhibitors as antiinflammatory agents can interfere with the disease process at 2 different stages, because they attenuate both early events of neutrophil activation and proteolytic tissue injury caused by released NSPs.

\section{Methods}

Mice. Simultaneous deficiency of the Prtn 3 and Ela2 gene cluster in $129 \mathrm{~S} 6 / \mathrm{SvEv}$ mice was generated by homologous recombination in embryonic stem cells as detailed in Supplemental Methods. Ela2 ${ }^{-/-}$mice on the $129 \mathrm{~S} 6 / \mathrm{SvEv}$ genetic background were previously generated (41) and were obtained as frozen embryos from the Medical Research Council Harwell (Oxford, United Kingdom). These and WT control mice were kept under pathogen-free conditions at the GSF Neuherberg (Munich, Germany). For most experiments, mice at 5-8 weeks of age were used. All animal experiments were performed with approval by the District Government of Upper Bavaria (Munich, Germany).

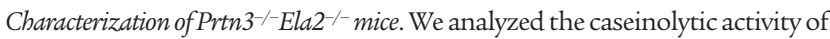
neutrophil lysates from WT Prtn $3^{+/+} \mathrm{Ela2}^{+/+}$mice, heterozygous $\mathrm{Prtn}^{+/-} \mathrm{Ela2}^{+/-}$ mice, and homozygous Prtn $3^{--}$Ela $2^{-/-}$mice by casein zymography. In short, $1.5 \mathrm{mg} / \mathrm{ml}$ casein (Sigma-Aldrich) was added to SDS polyacrylamide gels, and the lysate of $2.5 \times 10^{5}$ cells per well was separated under nonreducing conditions at $4^{\circ} \mathrm{C}$. Gels were then washed in PBS with $2.5 \%$ Triton X-100 followed by incubation overnight in Tris/ $\mathrm{HCl}$ (pH 7.4), $10 \mathrm{mM} \mathrm{CaCl}_{2}$, and $0.02 \%$ Brij 35 . Gels were washed, stained with Coomassie blue, and destained in acetic acid. 


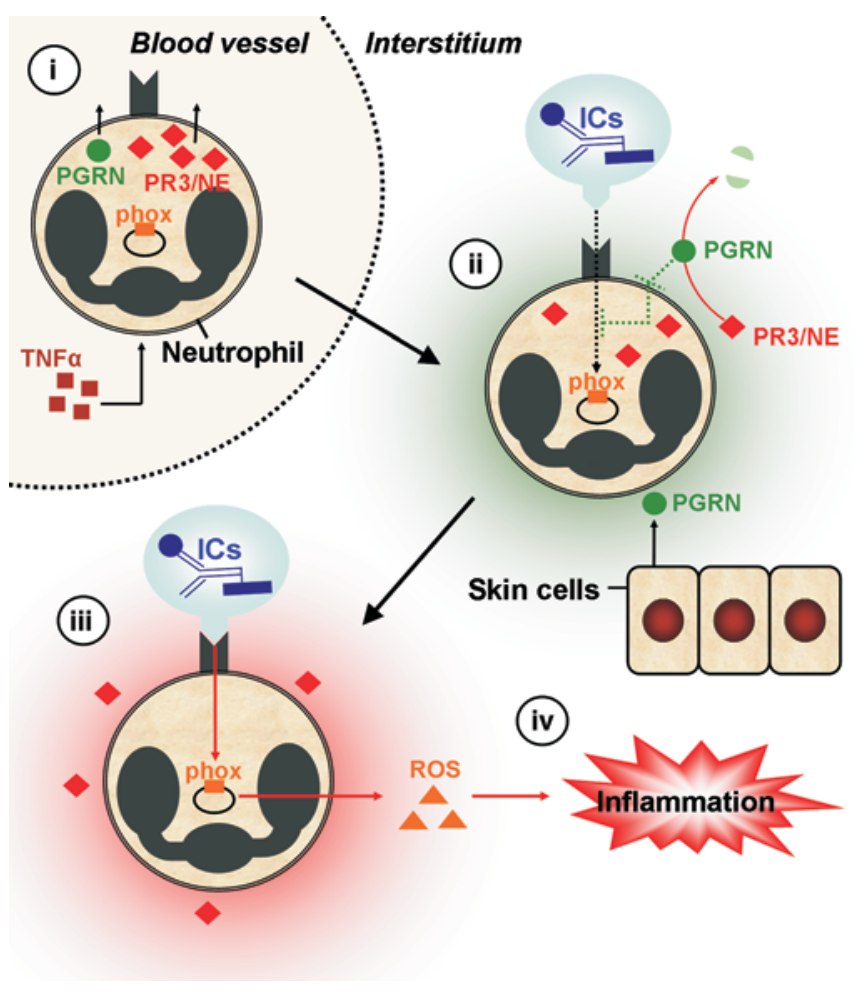

To confirm the successful and specific knockout of PR3 and NE, we performed Western blot analyses of bone marrow-derived neutrophils. After reducing SDS-PAGE of neutrophil lysates and electrotransfer onto nitrocellulose, we probed the membranes with polyclonal rabbit sera specific for the granule serine enzymes PR3, NE, CG, and MPO as previously described (42).

To control normal neutrophil differentiation in $\operatorname{Prtn}^{-/-E l a 2^{-/-}}$mice, peripheral blood neutrophils were characterized by flow cytometry using fluorescently labeled Gr-1- and CD11b-specific antibodies (BD Biosciences - Pharmingen) as previously described $(25,26)$. Blood smears were prepared and stained on glass slides according to the Diff-Quick protocol (Dade-Behring), and neutrophil morphology was analyzed by light microscopy. Differential blood leukocytes were identified by morphology and counted under the microscope to determine the percentage of each population. Moreover, the cell count per milliliter blood of each leukocyte population was determined using a hemocytometer (improved Neubauer chamber). For detailed descriptions of genetic characterization of $\mathrm{Prtn}^{-/-}$ Ela2 ${ }^{-/-}$mice, see Supplemental Methods.

Recombinant and purified proteins. Recombinant mouse PR3 was produced and purified as previously described (43). Human NE was purchased from Elastin Products. Recombinant human PGRN was prepared as previously described (21). Recombinant mouse PGRN (aa 18-589) was produced with an $\mathrm{N}$-terminal S-tag by transient expression into the supernatant of $\mathrm{H} 5$ insect cells using a modified pIEx-5 vector (Novagen; Merck) and the secretion signal of the adipokinetic hormone. Transfection of $\mathrm{H} 5$ insect cells cultured in TC-100 medium (Gibco; Invitrogen) was done using the Fugene HD transfection reagent (Roche) according to the manufacturer's instructions. At 6 days after transfection, the supernatant was harvested, and recombinant mouse PGRN was purified using an S-protein agarose column (Novagen; Merck). Following extensive washing of the column with PBS, the bound protein was eluted by $3 \mathrm{M}$ magnesium chloride. Immediately thereafter, fractions were dialyzed against PBS and analyzed by silver-stained SDS-PAGE. Pure fractions were pooled and dialyzed against $20 \mathrm{mM}$ ammonium bicar-

\section{Figure 7}

Proposed function of PR3 and NE in IC-mediated inflammation. TNF- $\alpha-$ primed neutrophils extravasate from blood vessels, translocate PR3/NE to the cellular surface, and discharge PGRN to the pericellular environment (i). During transmigration of interstitial tissues (ii), neutrophil activation is initially suppressed by relatively high pericellular levels of antiinflammatory PGRN (green shading), which is also produced locally by keratinocytes and epithelial cells of the skin. Until IC depots are reached, neutrophil activation is inhibited by PGRN. Surface receptors (e.g., Mac-1) recognize ICs, which results in signal transduction (black dotted arrow) and activation of the phox. The molecular pathway of PGRN-mediated inhibition is not completely understood, but it may interfere with integrin signaling after IC encounter (green dotted line inside the cell). Adherence of neutrophils to ICs (iii) further increases pericellular PR3 and NE activity. PR3 and NE cooperatively degrade PGRN in the early stage of neutrophilic activation to facilitate optimal neutrophil activation (red shading), resulting in sustained integrin signaling (red arrow) and robust production of ROS by the phox system. Subsequently, neutrophils release ROS together with other proinflammatory mediators and chemotactic agents, thereby enhancing the recruitment of further neutrophils and establishing inflammation (iv). In the absence of PR3/NE, the switch from inflammation-suppressing (ii) to inflammation-enhancing (iii) conditions is substantially delayed, resulting in diminished inflammation in response to ICs (iv).

bonate, lyophilized, and stored at $-80^{\circ} \mathrm{C}$ until use. For experiments, lyophilized protein was reconstituted in sterile PBS (Gibco; Invitrogen).

Skin inflammation models. Croton oil (Sigma-Aldrich) was diluted to $1 \%$ in acetone, and this dilution was topically applied to the ventral and the dorsal sides (20 $\mu \mathrm{l}$ each) of the ears of WT and $\operatorname{Prtn} 3^{-/-}$Ela $^{-/-}$mice $(n=4$ per group). After $4 \mathrm{~h}$, mice were sacrificed by $\mathrm{CO}_{2}$ inhalation. The earflap was split into 2 halves by carefully separating the dorsal and ventral skin. For histological analysis, dorsal and ventral halves of ears were subjected to wholemount immunostaining. After fixation in paraformaldehyde, ear halves were blocked with $1 \%$ BSA (PAA Laboratories) in PBS for $1 \mathrm{~h}$ at room temperature, probed with biotin-labeled anti-Gr-1 (RB6-8C5; BD Biosciences Pharmingen) to identify neutrophil granulocytes and anti-pan-laminin (L9393; Sigma-Aldrich) to visualize the EBM, diluted in 1\% BSA in PBS overnight at $4^{\circ} \mathrm{C}$ (while shaking), and washed with $1 \%$ BSA in PBS. Antibodies were detected with a repeated cycle of staining with anti-rabbit Alexa Fluor 488 (Invitrogen) and anti-rat Cy3 (Dianova) before tissue was embedded in elvanol and representative images taken with a Zeiss Axio Imager equipped with an ApoTome (Zeiss). Location of neutrophils was analyzed in detail for potential accumulation at the basement membrane. For quantification of neutrophil influx, at least 3 images of the inflamed skin were taken using a $\times 10$ objective to quantify total neutrophil infiltrates (calculated as the percentage of Gr-1 signal per microscopic field) using Metamorph software (Molecular Devices), while intravascular signal was excluded.

The RPA, a widely used model of IC-mediated inflammation, was induced in the skin of mice using OVA (grade V; Sigma-Aldrich) as the antigen and purified rabbit anti-OVA antibodies of the IgG class (Rockland Immunochemicals Inc.). Initially, the hair was removed from the ventral skin, and the area was cleaned with $70 \%$ alcohol. Anti-OVA $\operatorname{IgG}(2 \mu \mathrm{g} / \mu \mathrm{l})$ was deposited intradermally in a volume of $30 \mu \mathrm{l}$ using a 27 -gauge needle. Intradermal injection of $30 \mu \mathrm{l}$ unspecific polyclonal rabbit IgG $(2 \mu \mathrm{g} / \mu \mathrm{l}$; Sigma-Aldrich) served as a negative control in the same animal. Without delay, the antigen solution (OVA; $20 \mathrm{mg} / \mathrm{kg}$ body weight) was injected i.v. After $4 \mathrm{~h}$, mice were sacrificed by $\mathrm{CO}_{2}$ asphyxiation, and the inflammatory response was assessed by histology. Paraffin-embedded sections of the specimens were processed for $\mathrm{H} \& \mathrm{E}$ staining and analyzed by light microscopy for inflammatory cellular infiltrates. Neutrophils were additionally identified using Gr-1 immunohistochemistry. Briefly, deparaffinized tissue sections were incu- 
bated with a rat anti-Ly-6G antibody (BD Biosciences - Pharmingen) followed by a biotinylated goat anti-rat antibody (BioGenex). Bound antibodies were labeled with streptavidin-alkaline phosphatase and visualized with fast red (BioGenex). Sections were counterstained with Mayer hematoxylin solution (Merck Eurolab). Random high-power fields (HPFs) of the lesions were photographed using a Leica DFC320 CCD camera (Leica) attached to a Zeiss Axioplan 2 microscope (Zeiss) with a $\times 10$ objective. Digitized images were used to enumerate neutrophils as mean number per HPF in lesions from $\operatorname{WT}(n=12)$, Ela2 $2^{--}(n=13)$, and Prtn3 $3^{-/-}$Ela2 $^{-/-}$mice $(n=12)$.

To study the inhibitory capacity of PGRN on IC-stimulated inflammation, we deposited $2 \mu \mathrm{g}$ recombinant mouse PGRN by intradermal injection together with $30 \mu \mathrm{l}$ anti-OVA solution as described above. In the same mouse at different sites of the ventral skin, we intradermally applied antiOVA alone and control IgG as positive and negative controls, respectively. The RPA was started by i.v. administration of OVA. Mice were sacrificed $4 \mathrm{~h}$ after initiation, and biopsies were taken to histologically quantify neutrophil infiltrates per HPF in PGRN-treated and untreated lesions from WT and $\operatorname{Prtn} 3^{-/-}$Ela $2^{-/-}$mice ( $n=5$ per group).

Collagen gel chemotaxis assay. For migration assays, PureCol (INAMED) in $1 \times$ MEM (Sigma-Aldrich) and $0.4 \%$ sodium bicarbonate (Sigma-Aldrich) was mixed with cells in RPMI (Invitrogen) with 10\% FCS (Invitrogen) at a $2: 1$ ratio, resulting in gels with a collagen concentration of $1.6 \mathrm{mg} / \mathrm{ml}$. Neutrophils were primed by $10 \mathrm{ng} / \mathrm{ml}$ mouse TNF- $\alpha$ (Roche) for $15 \mathrm{~min}$ at $37^{\circ} \mathrm{C}$. Final cell concentrations in the assay were $1.6 \times 10^{6}$ granulocytes per milliliter of gel. Collagen-cell mixtures were cast in custom-made migration chambers with a thickness of $0.5-1.0 \mathrm{~mm}$. At $30 \mathrm{~min}$ after the assembly of the collagen fibers at $37^{\circ} \mathrm{C}$, the gel surface was covered with $50 \mu \mathrm{l}$ of $0.1 \mu \mathrm{g} / \mathrm{ml} \mathrm{C} 5 \mathrm{a}$ solution (R\&D Systems). Pictures of migrating cells were taken every $20 \mathrm{~s}$ using a Zeiss Axioplan 2 light-field microscope (Zeiss), and stacks of images were used to generate time-lapse videos in .AVI format using Metamorph (Molecular Devices). Chemotactic parameters were calculated and visualized as plots by analyzing the acquired data with a Chemotaxis and Migration Tool plug-in (http://www.ibidi.de/applications/ap_chemo.html).

Isolation of mouse neutrophils. Mouse neutrophils were purified from the bone marrow using a discontinuous percoll gradient (Amersham Bioscience) as previously described (44). Neutrophil preparations were characterized by Gr-1 and CD11b (Mac-1) double immunostaining using flow cytometry and were found to be at least $80 \%$ pure. To isolate murine neutrophils ex vivo from an inflammatory environment, we injected $1 \mathrm{ml}$ of a $9 \%$ sterile casein solution in PBS to induce peritonitis. At $4 \mathrm{~h}$ after injection, cells were harvested by peritoneal lavage using sterile PBS without $\mathrm{Ca}^{2+}$ or $\mathrm{Mg}^{2+}$ (Gibco; Invitrogen). Peritoneal cells were layered on a discontinuous histopaque 1119-1077 gradient (Sigma-Aldrich), and, after centrifugation for $30 \mathrm{~min}$ at $700 \mathrm{~g}$, neutrophils were isolated from the $1.119-1.077 \mathrm{~kg} / 1$ interphase. For anti-PGRN Western blots and PR3/NE activity assays, neutrophil lysates were prepared. In brief, neutrophils were lysed in $50 \mathrm{mM}$ Tris/ $\mathrm{HCl}$ ( $\mathrm{pH} 7.4$ ), $150 \mathrm{mM} \mathrm{NaCl}, 0.5 \mathrm{mM}$ EDTA, and $0.5 \%$ nonidet by mechanical disruption using syringes with 27-gauge needles. Cell debris was pelleted by centrifugation. All steps were carried out at $4^{\circ} \mathrm{C}$, and supernatant was frozen until used.

Neutrophil oxidative burst in vitro. To test neutrophil activation by ICs in vitro, we prepared immobilized ICs using an OVA/anti-OVA system as described previously (20). Isolated neutrophils were resuspended at a density of $2 \times 10^{6}$ cells $/ \mathrm{ml}$ in phenol red-free RPMI (Gibco; Invitrogen) containing $10 \mathrm{ng} / \mathrm{ml}$ mouse TNF- $\alpha$ (Roche) and added to immobilized ICs. Activation by $25 \mathrm{nM}$ PMA (Sigma-Aldrich) without TNF- $\alpha$ served as a positive control. We also determined the oxidative burst of neutrophils as a function of increasing PMA concentrations $(0.1-50 \mathrm{nM})$ in order to define the concentrations that yield submaximal responses in mouse neutrophils. ROS production as the readout for neutrophil activation was detected using dihydrorhodamine 6G (catalog no. D633; Invitrogen) according to the manufacturer's instructions, and the increase in fluorescence was measured over time with the fluorometer FLUOstar OPTIMA (BMG Labtech) at $37^{\circ} \mathrm{C}$. For each time point, the IC-specific oxidative burst was determined by subtracting the mean fluorescence read on negative control coating from that on IC coating. To compare independent experiments, we normalized oxidative burst relative to maximum ROS production by WT neutrophils.

To examine the effect of PGRN on neutrophil activation in vitro, we first approximated the concentration of cell-associated and secreted PGRN in the absence of PR3/NE during IC-mediated neutrophil stimulation by comparison with the positive control band in anti-PGRN Western blot, estimated to be approximately $100 \mathrm{nM}$. We then added recombinant PGRN at a final concentration of $100 \mathrm{nM}$ to isolated WT neutrophils stimulated by ICs or PMA. We compared the oxidative burst between PGRN-treated and untreated cells as described above.

Adhesion assay. Isolated neutrophils were labeled with Calcein AM (Invitrogen) and plated on ICs in the presence of $10 \mathrm{ng} / \mathrm{ml} \mathrm{TNF-} \alpha$ as previously described (20). In brief, wells were washed with PBS at the indicated time points, the number of adhering cells was determined using a fluorescence reader, and the percentage was calculated.

Phagocytosis assay. Neutrophil phagocytosis of opsonized, FITC-labeled E. coli was determined by flow cytometry using the Phagotest (Orpegen Phar$\mathrm{ma}$ ) according to the manufacturer's instructions, with some modifications. Briefly, $100 \mu$ l heparinized blood was incubated with $20 \mu$ FITC-labeled $E$. coli suspension $\left(10^{9}\right.$ bacteria $\left./ \mathrm{ml}\right)$ and incubated for $30 \mathrm{~min}$ at $37^{\circ} \mathrm{C}$. After washing and DNA staining (using DNA staining solution from the Phagotest kit), samples were analyzed by flow cytometry. Bacterial aggregates were excluded based on their lower DNA content, and neutrophil granulocytes were gated in the forward/side scatter analysis. Phagocytosis of FITC-labeled E. coli by neutrophil granulocytes was determined using the FL-1 channel regarding percentage as well as fluorescence intensity of phagocytosing cells.

Analysis of PGRN degradation. PGRN processing activity was tested with recombinant mouse PR3 and human NE each at a molar enzyme/substrate ratio of 1:10 according to the buffer conditions described by Zhu et al. (23). Recombinant human PGRN $(2.5 \mu \mathrm{g})$ was added to enzymes and incubated at $37^{\circ} \mathrm{C}$. At the time points indicated in the figures, samples were placed on ice, and the pattern of cleavage products was analyzed by reducing SDS-PAGE and subsequent silver staining.

To determine whether PGRN-degrading enzymes other than PR3 and NE existed in neutrophils, we incubated recombinant mouse PGRN with lysates from $3 \times 10^{4} \mathrm{WT}$, Ela2 $^{-/-}$, and Prtn3 $3^{-/-} \mathrm{Ela2}^{-{ }^{--}}$neutrophils for $1 \mathrm{~h}$ at $37^{\circ} \mathrm{C}$. Samples were separated by SDS-PAGE and transferred onto nitrocellulose membranes. After incubation for $2 \mathrm{~h}$ in blocking buffer $(5 \%$ fat-free dry milk in PBS/0.2\% Tween-20), mouse PGRN was detected by Western blotting with sheep anti-mouse PGRN antibody (R\&D Systems) at a concentration of $0.4 \mu \mathrm{g} / \mathrm{ml}$ and unspecific sheep IgG as a negative control $(1 \mu \mathrm{g} / \mathrm{ml}$; catalog no. I5131; Sigma-Aldrich). Bound antibodies were visualized using peroxidase-conjugated donkey anti-sheep Igs (1:10,000; Jackson ImmunoResearch Laboratories) and ECL reagents (Amersham Pharmacia). For actin detection, the membranes were incubated in a 1:5,000 dilution of anti-actin mouse monoclonal antibody (clone JLA-20; Calbiochem) followed by peroxidase-conjugated goat anti-mouse IgG and IgM incubation (1:10,000; Pierce) and subsequent ECL detection.

For detection of PGRN from neutrophils activated by ICs in vitro, we harvested the supernatant as well as the cellular pellet of isolated TNF- $\alpha-$ primed neutrophils after $3 \mathrm{~h}$ of IC stimulation. The concentrated supernatant as well as the cellular pellet of $2 \times 10^{5}$ IC-activated neutrophils was separated by reducing SDS-PAGE and subjected to anti-PGRN Western blotting as described above.

To analyze whether PGRN was cleaved by PR3/NE during inflammation in vivo, we harvested neutrophils from the inflamed peritoneum of WT 
and Prtn $3^{--}$Ela $2^{-/-}$mice. Lysates were prepared from these cells as described above, but in the presence of $75 \mu \mathrm{g} / \mathrm{ml}$ PMSF and protease inhibitor cocktail (Calbiochem). Total lysates from $8 \times 10^{5}$ cells were subjected to antiPGRN Western blotting, which was carried out as described above.

Statistics. All results are given as mean and SEM for data derived from different in vivo experiments and as mean and SD for data resulting from triplicate in vitro assays using isolated cells or cell lysates. Normal distribution of data sets was determined by the Kolmogorov-Smirnov test before we applied an unpaired Student's $t$ test to compare 2 groups. Calculations were done using GraphPad Prism software. A $P$ value less than 0.05 was considered statistically significant.

\section{Acknowledgments}

We thank Bettina Maar, Martin Skerhut, and Elisabeth Stegmann for excellent technical assistance and breeding of mice. Further- more, we are grateful to Hartmut Wekerle and Edgar Meinl for constant interest and helpful suggestions. This work was supported by grants from the Bundesministerium für Bildung und Forschung (01GC0104) and the Deutsche Forschungsgemeinschaft (SFB 571, subproject A4, and SFB 469, subproject B7).

Received for publication December 6, 2007, and accepted in revised form May 14, 2008.

Address correspondence to: Kai Kessenbrock or Dieter E. Jenne, Max-Planck-Institute of Neurobiology, Department of Neuroimmunology, Am Klopferspitz 18, 82152 Martinsried, Germany. Phone: 49-89-8578-3588; Fax: 49-89-8578-3790; E-mail: kessenbrock@neuro.mpg.de (K. Kessenbrock); djenne@neuro. mpg.de (D.E. Jenne).
1. Nathan, C. 2006. Neutrophils and immunity: challenges and opportunities. Nat. Rev. Immunol. 6:173-182.

2. Jancar, S., and Sanchez, C.M. 2005. Immune complex-mediated tissue injury: a multistep paradigm. Trends Immunol. 26:48-55

3. Ravetch, J.V. 1994. Fc receptors: rubor redux. Cell. 78:553-560.

4. Belaaouaj, A., et al. 1998. Mice lacking neutrophil elastase reveal impaired host defense against gram negative bacterial sepsis. Nat. Med. 4:615-618.

5. Belaaouaj, A., Kim, K.S., and Shapiro, S.D. 2000. Degradation of outer membrane protein A in Escherichia coli killing by neutrophil elastase. Science. 289:1185-1188.

6. Reeves, E.P., et al. 2002. Killing activity of neutrophils is mediated through activation of proteases by K+ flux. Nature. 416:291-297.

7. Segal, A.W. 2005. How neutrophils kill microbes. Annu. Rev. Immunol. 23:197-223.

8. Weinrauch, Y., Drujan, D., Shapiro, S.D., Weiss, J., and Zychlinsky, A. 2002. Neutrophil elastase targets virulence factors of enterobacteria. Nature. 417:91-94.

9. El Ouriaghli, F., et al. 2003. Neutrophil elastase enzymatically antagonizes the in vitro action of G-CSF: implications for the regulation of granulopoiesis. Blood. 101:1752-1758.

10. Skold, S., Rosberg, B., Gullberg, U., and Olofsson, T. 1999. A secreted proform of neutrophil proteinase 3 regulates the proliferation of granulopoietic progenitor cells. Blood. 93:849-856.

11. Bories, D., Raynal, M.C., Solomon, D.H., Darzynkiewicz, Z., and Cayre, Y.E. 1989. Down-regulation of a serine protease, myeloblastin, causes growth arrest and differentiation of promyelocytic leukemia cells. Cell. 59:959-968.

12. Campbell, E.J., Campbell, M.A., and Owen, C.A. 2000. Bioactive proteinase 3 on the cell surface of human neutrophils: Quantification, catalytic activity, and susceptibility to inhibition. J. Immunol. 165:3366-3374.

13. Owen, C.A., Campbell, M.A., Sannes, P.L., Boukedes, S.S., and Campbell, E.J. 1995. Cell surface-bound elastase and cathepsin-G on human neutrophils - a novel, nonoxidative mechanism by which neutrophils focus and preserve catalytic activity of serine proteinases. J. Cell Biol. 131:775-789.

14. Shapiro, S.D. 2002. Neutrophil elastase - Path clearer, pathogen killer, or just pathologic? Am. J. Respir. Cell Mol. Biol. 26:266-268.

15. Carden, D.L., and Korthuis, R.J. 1996. Protease inhibition attenuates microvascular dysfunction in postischemic skeletal muscle. Am. J. Physiol. 271:H1947-H1952.

16. Hirche, T.O., Atkinson, J.J., Bahr, S., and Belaaouaj, A. 2004. Deficiency in neutrophil elastase does not impair neutrophil recruitment to inflamed sites. Am. J. Respir. Cell Mol. Biol. 30:576-584.

17. Young, R.E., et al. 2004. Neutrophil elastase (NE)deficient mice demonstrate a nonredundant role for NE in neutrophil migration, generation of proinflammatory mediators, and phagocytosis in response to zymosan particles in vivo. J. Immunol. 172:4493-4502.

18. Pham, C.T. 2006. Neutrophil serine proteases: specific regulators of inflammation. Nat. Rev. Immunol. 6:541-550.

19. Adkison, A.M., Raptis, S.Z., Kelley, D.G., and Pham, C.T. 2002. Dipeptidyl peptidase I activates neutrophil-derived serine proteases and regulates the development of acute experimental arthritis. J. Clin. Invest. 109:363-371.

20. Raptis, S.Z., Shapiro, S.D., Simmons, P.M., Cheng, A.M., and Pham, C.T. 2005. Serine protease cathepsin $\mathrm{G}$ regulates adhesion-dependent neutrophil effector functions by modulating integrin clustering. Immunity. 22:679-691.

21. He, Z., Ong, C.H., Halper, J., and Bateman, A. 2003. Progranulin is a mediator of the wound response. Nat. Med. 9:225-229.

22. He, Z., and Bateman, A. 2003. Progranulin (granulin-epithelin precursor, PC-cell-derived growth factor, acrogranin) mediates tissue repair and tumorigenesis. J. Mol. Med. 81:600-612.

23. Zhu, J., et al. 2002. Conversion of proepithelin to epithelins: roles of SLPI and elastase in host defense and wound repair. Cell. 111:867-878.

24. Bateman, A., Belcourt, D., Bennett, H., Lazure, C., and Solomon, S. 1990. Granulins, a novel class of peptide from leukocytes. Biochem. Biophys. Res. Commun. 173:1161-1168.

25. Fleming, T.J., Fleming, M.L., and Malek, T.R. 1993. Selective expression of Ly-6G on myeloid lineage cells in mouse bone-marrow - Rb6-8C5 Mab to granulocyte-differentiation antigen (Gr-1) detects members of the Ly-6 family. J. Immunol. 151:2399-2408.

26. Hestdal, K., et al. 1991. Characterization and regulation of Rb6-8C5 antigen expression on murine bone-marrow cells. J. Immunol. 147:22-28.

27. Arthus, M., and Breton, M. 1903. Cutaneous lesions produced by injections of horse serum in a rabbit anaphylactised by and for this serum. C. R. Seances Soc. Biol. Fil. 55:1478-1480.

28. Wang, S., et al. 2006. Venular basement membranes contain specific matrix protein low expression regions that act as exit points for emigrating neutrophils. J. Exp. Med. 203:1519-1532.

29. Lin, W.L., Zehr, C., Lewis, J., and Dickson, D.W. 2007. Progranulin is located in secretory granules and vesicles of neutrophils and macrophages by immunogold electron microscopy. FASEBJ. 21:A22.

30. Gonzalez, E.M., Mongiat, M., Slater, S.J., Baffa,
R., and Iozzo, R.V. 2003. A novel interaction between perlecan protein core and progranulin - Potential effects on tumor growth. J. Biol. Chem. 278:38113-38116.

31. Campbell, E.J., and Owen, C.A. 2007. The sulfate groups of chondroitin sulfate- and heparan sulfate-containing proteoglycans in neutrophil plasma membranes are novel binding sites for human leukocyte elastase and cathepsin G. J. Biol. Chem. 282:14645-14654.

32. Locksley, R.M., Wilson, C.B., and Klebanoff, S.J. 1983. Increased respiratory burst in myeloperoxidase-deficient monocytes. Blood. 62:902-909.

33. Rosen, H., and Klebanoff, S.J. 1976. Chemiluminescence and superoxide production by myeloperoxidase-deficient leukocytes. J. Clin. Invest. 58:50-60.

34. Couto, M.A., Harwig, S.S., Cullor, J.S., Hughes, J.P., and Lehrer, R.I. 1992. eNAP-2, a novel cysteine-rich bactericidal peptide from equine leukocytes. Infect. Immun. 60:5042-5047.

35. Shapiro, S.D., et al. 2003. Neutrophil elastase contributes to cigarette smoke-induced emphysema in mice. Am. J. Pathol. 163:2329-2335.

36. Liu, Z., et al. 2000. The serpin alpha 1-proteinase inhibitor is a critical substrate for gelatinase B/ MMP-9 in vivo. Cell. 102:647-655.

37. Liu, Z., et al. 2000. A critical role for neutrophil elastase in experimental bullous pemphigoid. J. Clin. Invest. 105:113-123.

38. Stoller, J.K., and Aboussouan, L.S. 2005. alpha 1antitrypsin deficiency. Lancet. 365:2225-2236.

39. Benarafa, C., Priebe, G.P., and Remold-O'Donnell, E. 2007. The neutrophil serine protease inhibitor serpinb1 preserves lung defense functions in Pseudomonas aeruginosa infection. J. Exp. Med. 204:1901-1909.

40. Ashcroft, G.S., et al. 2000. Secretory leukocyte protease inhibitor mediates non-redundant functions necessary for normal wound healing. Nat. Med. 6:1147-1153.

41. Tkalcevic, J., et al. 2000. Impaired immunity and enhanced resistance to endotoxin in the absence of neutrophil elastase and cathepsin G. Immunity. 12:201-210.

42. Hirche, T.O., Gaut, J.P., Heinecke, J.W., and Belaaouaj, A. 2005. Myeloperoxidase plays critical roles in killing Klebsiella pneumoniae and inactivating neutrophil elastase: Effects on host defense. J. Immunol. 174:1557-1565.

43. Pfister, H., et al. 2004. Antineutrophil cytoplasmic autoantibodies against the murine homolog of proteinase 3 (Wegener autoantigen) are pathogenic in vivo. Blood. 104:1411-1418.

44. Lowell, C.A., Fumagalli, L., and Berton, G. 1996. Deficiency of Src family kinases p59/61(hck) and p58(c-fgr) results in defective adhesion-dependent neutrophil functions. J. Cell Biol. 133:895-910. 